\title{
Functional inks of graphene, metal dichalcogenides and black phosphorous for photonics and (opto)electronics
}

\author{
Richard C. T. Howe ${ }^{a}$, Guohua $\mathrm{Hu}^{a}$, Zongyin Yang ${ }^{a}$, Tawfique Hasan ${ }^{a, *}$ \\ ${ }^{a}$ Cambridge Graphene Centre, 9 JJ Thomson Avenue, Cambridge, UK
}

\begin{abstract}
We discuss the emerging role of solution processing and functional ink formulation in the fabrication of devices based on two dimensional (2d) materials. By drawing on examples from our research, we show that these inks allow $2 \mathrm{~d}$ materials to be exploited in a wide variety of applications, including in photonics and (opto)electronics.
\end{abstract}

Keywords: Graphene, Transition Metal Dichalcogenides, TMDs, Black Phosphorous, Phosphorene, Functional Inks, 2d Materials, Inkjet Printing, Ultrafast Lasers, Flexible Electronics

\section{INTRODUCTION}

Layered materials (LMs) such as graphite have a crystal structure consisting of covalently bonded 2-dimensional (2d) planes, bound into a stack via weak van der Waals (vdW) forces. Atomically thin layers can be exfoliated from these bulk LM crystals, or grown directly, to yield $2 \mathrm{~d}$ or quasi-2d ( 1-10 layers) materials. The class of $2 \mathrm{~d}$ materials is currently of intense research interest across many fields due to their remarkable properties. ${ }^{1-4}$ Although their low dimensionality results in the emergence of these exotic properties, one of the key challenges at present is to develop techniques to translate these headline results, typically measured on one-off devices requiring complex fabrication, into real-world systems. One such route is through the formulation of $2 \mathrm{~d}$ material inks which, when combined with established printing techniques, can be used to reliably deposit functional coatings for large area devices. The most commonly studied $2 \mathrm{~d}$ materials at present include graphene, transition metal dichalcogenides (TMDs) and black phosphorus (BP). In the course of this article, we will briefly discuss key properties and production techniques of these materials, before presenting a range of application examples from our research.

\section{PROPERTIES AND PRODUCTION OF 2D MATERIALS}

\subsection{Graphene}

Among the $2 \mathrm{~d}$ material family, graphene, consisting of an atomically thin layer of hexagonally arranged carbon atoms (Fig. 1), is the most widely studied. ${ }^{1,5,6}$ In 1999, Ref. 7 reported that highly orientated pyrolytic graphite (HOPG) patterned with raised "islands" could be cleaved by rubbing it on silicon, yielding few-layer graphene flakes. The authors noted at the time that it should be possible to extend this technique to produce monolayer flakes, which was subsequently realized in $2004 .{ }^{7}$ Graphene has many key properties that make it well-suited to flexible and transparent electronics. ${ }^{1,5,6}$ While undoped graphene has a relatively high intrinsic sheet resistance of $6 \mathrm{k} \Omega / \mathrm{sq}^{6}{ }^{6}$ in practice, graphene samples always show some level of doping, and hence lower sheet resistance. Ref. 1 derived a relationship between sheet resistance and DC conductivity for single and few layer graphene:

$$
R_{s}=(n \mu e N)^{-1}
$$

where $n$ is the number of carriers $\left(\mathrm{cm}^{-2}\right), \mu$ is the carrier mobility $\left(\mathrm{cm}^{2} \mathrm{~V}^{-1} \mathrm{~s}^{-1}\right), e$ is electron charge $(1.6 \mathrm{x}$ $10^{-19} \mathrm{C}$ ), and $\mathrm{N}$ is the number of layers. When combined with the typical values of $n$ and $\mu$ for CVD samples $\left(10^{12}-10^{13} \mathrm{~cm}^{-2}\right.$ and $1000-20000 \mathrm{~cm}^{2} \mathrm{~V}^{-1} \mathrm{~s}^{-1}$ respectively), sheet resistances as low as $\sim 30 \Omega /$ sq can be achieved from monolayer graphene, with even lower sheet resistance from bilayer and few-layer samples. Meanwhile, graphene also has a high optical transparency $(\sim 97.7 \%)$ from the visible to the near infrared. ${ }^{8,9}$ The absorption

\footnotetext{
*Further author information: (Send correspondence to T.H.)

T.H.: E-mail: th270@cam.ac.uk, Telephone: +44 1223748362
} 


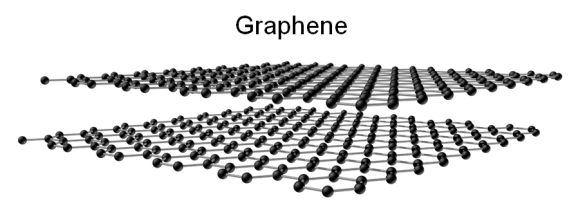

Zero-gap material

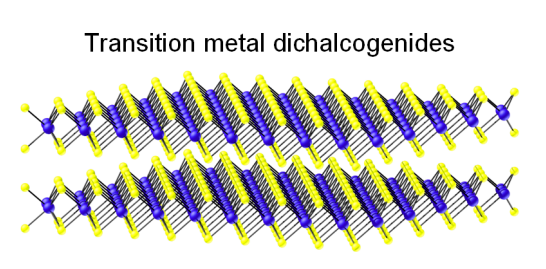

Metallic (e.g. $\mathrm{NbS}_{2}$ ), semiconducting (e.g. $\mathrm{MoS}_{2}$ )

or insulating (e.g. $\mathrm{HfS}_{2}$ )

$\mathrm{MoS}_{2} \mathrm{ML}-1.8 \mathrm{eV}$ direct bandgap

$\mathrm{MoS}_{2}$ Bulk- $1.2 \mathrm{eV}$ indirect bandgap

$\mathrm{WS}_{2} \mathrm{ML}-1.9 \mathrm{eV}$ direct bandgap

$\mathrm{WS}_{2}$ Bulk- $1.4 \mathrm{eV}$ indirect bandgap

$\mathrm{MoSe}_{2} \mathrm{ML}-1.5 \mathrm{eV}$ direct bandgap

$\mathrm{MoSe}_{2}$ Bulk- $1.1 \mathrm{eV}$ indirect bandgap

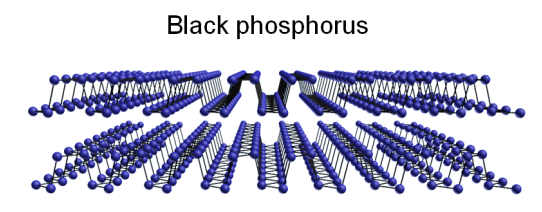

Direct bandgap semiconductor

$\mathrm{ML}-1.5 \mathrm{eV}$ direct bandgap

Bulk - 0.3 eV direct bandgap

Figure 1. Structures and electrical properties of some key $2 \mathrm{~d}$ materials.

spectrum is largely featureless throughout this region due to the linear dispersion of Dirac electrons in the graphene band-structure, aside from a peak in the UV region, a signature of the van Hove singularity in its density of states. ${ }^{10}$ It is also capable of sustaining $~ 25 \%$ strain without breaking. ${ }^{11}$ This combination of factors suggest that graphene is an attractive material for (opto)electronic applications such as flexible transparent conducting electrodes, where it may exceed the performance of the currently dominant material (indium tin oxide - ITO), not only in terms of transmittance and sheet resistance, but also in cost and flexibility. ${ }^{1,12}$

\subsection{Transition metal dichalcogenides}

Looking beyond graphene, one of the largest groups of $2 \mathrm{~d}$ materials is TMDs. They have the general formula $\mathrm{MX}_{2}$, where $\mathrm{M}$ is a transition metal such as molybdenum (Mo) or tungsten (W), and $\mathrm{X}$ is a group VI element such as sulfur (S), selenium (Se) or tellurium (Te). Their structure consists of a layer of metal atoms covalently bonded between two layers of chalcogen atoms (Fig. 1). Depending on the metal and chalcogen, as well as the atomic coordination, TMDs may be metallic (e.g. $\mathrm{NbS}_{2}$ ), semiconducting (e.g. $\left.\mathrm{MoS}_{2}\right)$, or insulating (e.g. $\left.\mathrm{HfS}_{2}\right){ }^{2,3,13}$ For electronic, optoelectronic, and photonic applications, semiconducting TMDs (s-TMDs) are of particular interest because of their layer-dependent bandgap. As a zero-gap material, graphene is unsuitable for digital electronics using conventional device architecture, giving small $(\sim 2-10)$ ON-OFF ratios (i.e. the ratio of current in the ON and $\mathrm{OFF}$ states). ${ }^{14}$ In contrast, the bandgap of s-TMDs makes them attractive for field-effect transistors. ${ }^{15-19}$ While the electron mobilities in these devices are lower than graphene $\left(\sim 10-50 \mathrm{~cm}^{2} \mathrm{~V}^{-1} \mathrm{~s}^{-1}\right),{ }^{17,20}$ the higher ON-OFF ratios $\left(>10^{6}\right)^{15,16,18,19}$ allow for efficient switching and device operation. Bulk s-TMDs typically have indirect bandgaps. ${ }^{13}$ Changes in the band-structure with reducing layer count means that they progressively shift, ultimately becoming direct bandgaps in monolayer samples. ${ }^{2,3,21,22}$ For example, Ref. 22 estimated the bandgap for few-layer $\mathrm{MoS}_{2}$ with layer counts from the lowest energy photoluminescence peak, finding that the bandgap shifted from $1.29 \mathrm{eV}$ for bulk $\mathrm{MoS}_{2},{ }^{13}$ to $\sim 1.35 \mathrm{eV}$ for 6 layers and to $\sim 1.80 \mathrm{eV}$ for monolayers. ${ }^{21}$ Collectively, s-TMDs have a range of bandgaps spanning the visible and near infra-red spectrum, making them well-suited to applications such as light emission ${ }^{23}$ and photodetection, ${ }^{24-26}$ as well as more generally for (opto)electronics and photonics.

We note that experimental studies on few-layer s-TMDs predate those on graphene, with reports of few-layer $\mathrm{MoS}_{2}$ dating back to 1963, when R. Frindt and A. Yoffe reported new features in the absorption spectra of 
exfoliated $\mathrm{MoS}_{2} \cdot{ }^{27}$ However, limitations with the characterization and processing techniques available at the time meant that these materials were not exploited until more recently, when the discovery of graphene led to a renewed interest in $2 \mathrm{~d}$ materials.

\subsection{Black phosphorus}

$\mathrm{BP}$, or phosphorene, has recently emerged as another $2 \mathrm{~d}$ material of interest, ${ }^{4}$ in particular for optoelectronic applications. This is because it is a direct band-gap semiconductor in mono- and few-layer, as well as in bulk. ${ }^{28,29}$ As with s-TMDs, the bandgap varies with number of layers, from $\sim 0.3 \mathrm{eV}$ for bulk material to $\sim 1.5 \mathrm{eV}$ for monolayers, presenting exciting opportunities for electronics and optoelectronics in the near infrared region. ${ }^{30-32}$ The structure, (Fig. 1) consists of "wrinkled" layers of phosphorus atoms. Research into BP is limited to some extent at present due to its poor environmental stability, as it rapidly degrades via oxidation in the presence of either water or oxygen..$^{30,32,33}$ However, various strategies have recently been developed to isolate BP flakes from atmospheric conditions, including encapsulation ${ }^{33}$ and solution processing in anhydrous solvents, ${ }^{30}$ allowing demonstration of BP-based transistors ${ }^{31,33}$ and photodetectors. ${ }^{34}$

\subsection{Other layered materials}

More recently, many other LMs have attracted interest, as they offer complementary properties. These include materials such as $\mathrm{Bi}_{2} \mathrm{Te}_{3}$ and $\mathrm{Bi}_{2} \mathrm{Se}_{3}$, which are topological insulators in their bulk form, ${ }^{35}$ and MXenes (early transition metal carbides and carbonitrides) such as $\mathrm{Ti}_{3} \mathrm{C}_{2}$ and $\mathrm{Ti}_{3} \mathrm{CN}$, which are promising anode materials for energy storage applications. ${ }^{36,37}$ Additionally, many clays and minerals have layered crystal structures. ${ }^{38}$ As the majority of them are insulating, they have primarily been used to enhance the strength of composites and as barrier materials rather than for their optoelectronic properties. ${ }^{38}$ We will now discuss ways in which these materials can be produced with a view to realizing their potential.

\subsection{2d material production techniques}

Mono- and few-layer flakes and films of $2 \mathrm{~d}$ materials can be produced by both top-down methods such as exfoliation, and bottom-up methods such as chemical vapor deposition (CVD). Exfoliation is possible due to the relatively weak vdW forces between the layers compared to the strong intralayer covalent bonds. For fundamental materials research, micromechanical cleavage, where a bulk $2 \mathrm{~d}$ material is repeatedly cleaved with an adhesive tape, remains the dominant method. ${ }^{39}$ However, while the quality of exfoliated crystal is very high, this strategy has extremely low yield, and cannot be readily scaled up, ${ }^{39}$ rendering it unsuitable for large-scale device manufacture.

For large area applications, two key techniques have emerged. The first is growth via CVD. ${ }^{12,39-43}$ While the specifics of the technique vary between materials, the underlying principles are the same. One or more vaporized or gaseous precursors is passed over a substrate at an elevated temperature (typically 600 - 1000 $\left.{ }^{\circ} \mathrm{C}\right),{ }^{12,39-43}$ catalyzing reactions between the precursors, and leading to the growth of a mono- or few-layer film. For growth of graphene, the substrate is typically copper ${ }^{12}$ or nickel, ${ }^{43}$ while s-TMDs are more usually grown on substrates such as silicon ${ }^{40,41,44}$ or sapphire. ${ }^{41,45}$ The choice of suitable substrates for CVD is limited, meaning that lengthy and complex multi-step processes are required to transfer the material from the growth substrate to the device substrate. ${ }^{12,39,41,44-48}$ A typical transfer process involves coating with a polymer such as polymethyl methacrylate (PMMA), ${ }^{41,44,46-48}$ or attaching a thermal release tape ${ }^{12}$ (i.e. tape that loses its adhesiveness when heated) then etching the growth substrate. The $2 \mathrm{~d}$ material and the support polymer/tape can then be deposited onto the target substrate, before the polymer is dissolved (or the tape is heated and removed) to leave the isolated $2 \mathrm{~d}$ film. ${ }^{12,41,44,46-48}$ In addition to the relative complexity, transferring can also introduce defects, either resulting from chemical or mechanical damage during the etching and transfer process, ${ }^{47}$ or residual polymer ${ }^{49,50}$ or adhesive ${ }^{12}$ in the finished film. Along with the high production temperature, this can restrict the use of CVD for many low-cost, large area and high throughput applications, in particular, on flexible substrates.

Solution-based exfoliation techniques have recently attracted significant interest. This is because this approach allows large-scale production of $2 \mathrm{~d}$ material dispersions under ambient conditions. ${ }^{39,51-53}$ By avoiding the need for high temperature and sacrificial substrates, the complexity and cost of manufacture is significantly 
reduced compared to CVD. More importantly, these dispersions can be formulated into inks for a variety of established printing and coating techniques, ${ }^{39,54,55}$ or blended with polymers to fabricate composite materials. ${ }^{56-61}$ The exfoliated materials can be easily doped or decorated with functional groups, enabling new material functionality. Solution processing thus opens up a wide variety of opportunities for materials processing, deposition and device fabrication for large volume manufacturing at a fraction of the cost of other production techniques.

\section{LM SOLUTION PROCESSING}

Solution-based exfoliation techniques rely on overcoming the interlayer vdW forces, exfoliating bulk LM crystals to give dispersed few- and mono-layer flakes. One of the earliest approaches to solution-based exfoliation was through intercalation. ${ }^{39,62,63}$ This is typically achieved by immersing bulk LM in a solution containing lithium compounds. ${ }^{63,64}$ The lithium atoms intercalate between the LM planes, increasing the separation between the layers and thus reducing the vdW attraction forces between them. Lithium intercalated compounds are particularly well-suited to exfoliation in water via stirring or sonication, as the hydrogen released when lithium is exposed to water promotes the exfoliation process. ${ }^{62}$ However, intercalation can lead to structural changes in the exfoliated materials, altering their properties. For example, $\mathrm{MoS}_{2}$ exfoliated via intercalation typically forms the unstable metallic $1 \mathrm{~T}$ phase, requiring annealing at high temperature $\left(e . g . \sim 300^{\circ} \mathrm{C}\right)$ to restore the semiconducting $2 \mathrm{H}$ phase of pristine material. ${ }^{64}$

A variation on the intercalation process is electrochemical exfoliation where a LM bulk crystal is used as the working electrode in an electrochemical cell. When a positive voltage is applied to the electrode, negative ions from the liquid intercalate between the layers, leading to exfoliation. The process has been demonstrated with s-TMDs ${ }^{65}$ and graphene. ${ }^{66,67}$ As with the intercalation process discussed above, the exfoliated material is altered compared to pristine material. ${ }^{65-67}$

An alternative to intercalation is chemical treatment of the bulk material, with oxidation of graphite to graphite oxide via variants of the Hummers method being a prime example. ${ }^{68,69}$ Graphene oxide (GO) is hydrophilic due to functional groups introduced on the basal plane, and has larger interlayer spacing, allowing exfoliation in water. ${ }^{67,69-71}$ However, GO is electrically insulating. ${ }^{68,70}$ Reduction to reduced graphene oxide (rGO) is possible via thermal or chemical treatment. ${ }^{70,71}$ Nevertheless, rGO is fundamentally altered by the oxidation-reduction process and the properties of pristine graphene cannot be completely restored. ${ }^{68}$

It is also possible to exfoliate LMs without the need for chemical pre-treatment, through techniques such as ultrasound assisted liquid phase exfoliation (UALPE), ${ }^{51-53,56}$ and high shear mixing (including high pressure homogenization). ${ }^{72}$ In both these strategies, the solvent is selected such that the enthalpy of exfoliation (i.e. the energy cost from exposing additional LM surface area to the solvent) is minimized. ${ }^{53}$ Both techniques then exploit high shear forces to overcome the weak interlayer vdW forces, either through the collapse of microbubbles ${ }^{73}$ (in the case of the former), or through the mixing process itself ${ }^{72}$ (in the case of the latter). It has been shown that Hansen solubility parameters (HSPs), which divide the solvent-LM interactions into dispersive, polar, and

hydrogen bonding components, can aid solvent selection for the exfoliation process. ${ }^{53,74}$ Solvents with HSPs matching those experimentally derived for LMs are able to form stable dispersions. ${ }^{53,74}$ However, many of the best suited solvents (e.g. N-methylpyrrolidone - NMP, cyclohexanone, N-Cyclohexyl-2-pyrrolidone - CHP) ${ }^{51,53}$ have high boiling points (NMP $\sim 200^{\circ} \mathrm{C}$, cyclohexanone $\sim 155^{\circ} \mathrm{C}, \mathrm{CHP} \sim 154^{\circ} \mathrm{C}$ ), leading to challenges in printing and processing. For many integration strategies, especially printing, coating and composites, exfoliation in low boiling points such as alcohols and water is desirable. In this case, the mismatch in HSPs is too great to achieve stable dispersion in the solvents alone (although meta-stable dispersion is possible in some alcohols). ${ }^{53,74}$ Exfoliation in these less favorable solvents is possible through the introduction of dispersants such as polymers and surfactants, which can stabilize exfoliated flakes via steric and Coulomb repulsion. ${ }^{53}$ In the case of BP, there is an additional consideration related to the material stability in the presence of moisture and oxygen, both while in dispersion, as well as in any subsequent processing steps. ${ }^{30}$ Ref. 31 showed that exfoliation into anhydrous solvents such as CHP allows BP stability to be maintained while in solution. Following deposition, the flakes degraded over the course of a few days due to oxidation at the flake edges. ${ }^{30}$ By adding a stabilizing polymer to an exfoliation solvent, we have recently found that this stability can be greatly extended (several weeks), allowing BP dispersions to be printed or coated without the need for a controlled atmosphere. 


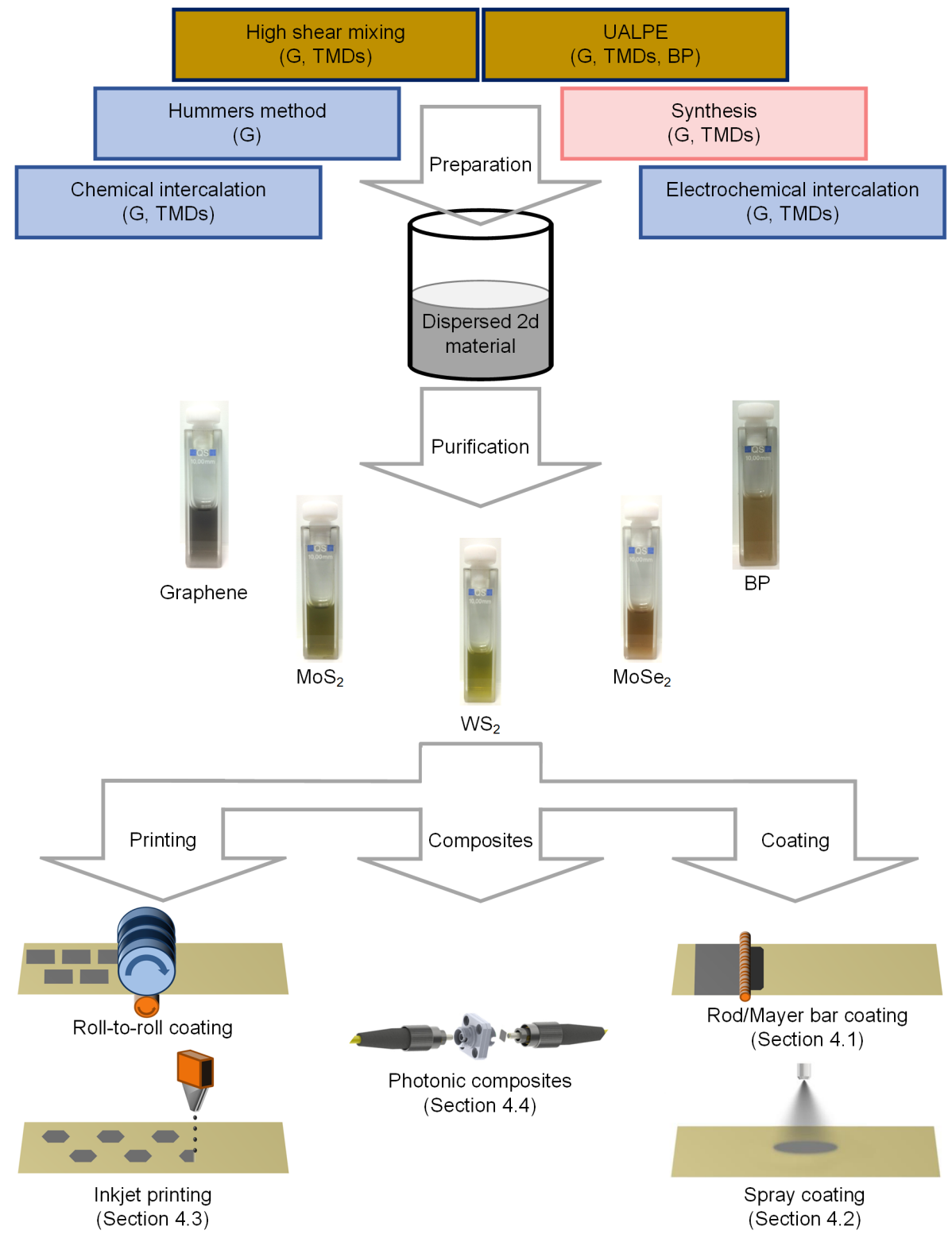

Figure 2. Summary of common solution processing and deposition techniques of $2 \mathrm{~d}$ materials. The processing techniques are divided into top-down without chemical treatment (orange, horizontal hatch), top-down with chemical pre-treatment (green, vertical hatch) and bottom-up (blue, cross hatch). The letters indicate the techniques that have been demonstrated for each material to date: G - Graphene, TMD - Transition Metal Dichalcogenides, BP - Black phosphorus.

Finally, LM nanosheets can also be produced by bottom-up synthesis, which has been demonstrated both with graphene ${ }^{75,76}$ and s-TMDs. ${ }^{77-79}$ However, research into these methods remains at an early stage and the growth processes remain poorly understood. At present, therefore, top-down methods remain the most common solution processing routes for large-scale $2 \mathrm{~d}$ material production.

\section{APPLICATIONS FOR 2D MATERIAL INKS AND COMPOSITES}

Aside from the advantages of high yield and low processing temperature, the key benefit to solution-processed $2 \mathrm{~d}$ materials is the variety of methods they can be processed, deposited and integrated for various applications 
(Fig. 2). For example, they can be formulated into inks to be used in well-established coating systems such as spray-coating, as well as printing techniques such as inkjet-printing, ${ }^{26,54,55,80}$ along with higher throughput methods such as flexography (a roll-to-roll printing process capable of printing at $>100 \mathrm{~m} / \mathrm{minute}$ ). When appropriately formulated, the inks can be used even without modification of a commercial printer, allowing $2 \mathrm{~d}$ materials to be deposited with conventional graphics presses rather than specialist equipment. This is particularly important as the use of printing for economic and mass production of electronic devices, components and subsystems is a rapidly growing field. The scalable manufacturability of functional inks will allow $2 \mathrm{~d}$ materials to be introduced into these applications. Aside from printing, $2 \mathrm{~d}$ materials can also be blended with polymers to fabricate composites for applications such as ultrafast photonics. ${ }^{56-60,81,82}$ We now present examples from our research to demonstrate some of the applications of graphene and $2 \mathrm{~d}$ material inks and composites.

\subsection{Hybrid graphene inks for transparent conducting electrodes}
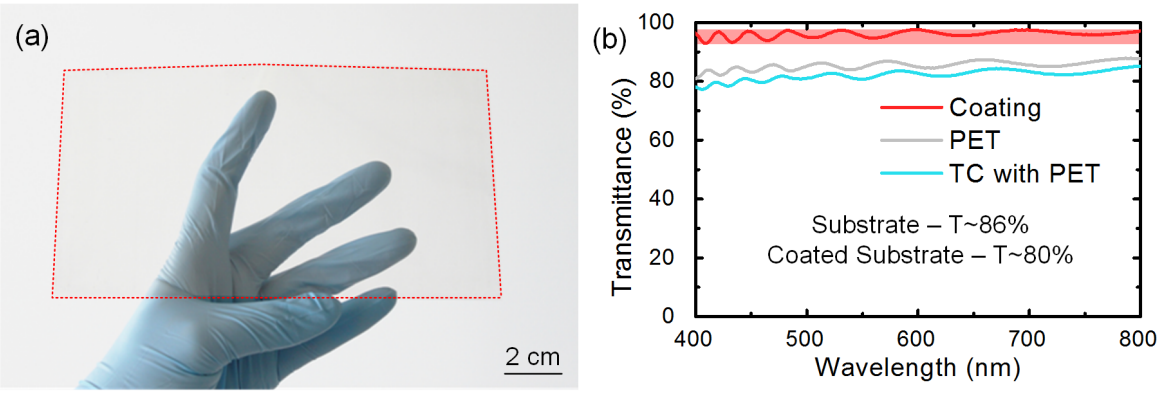

(c)

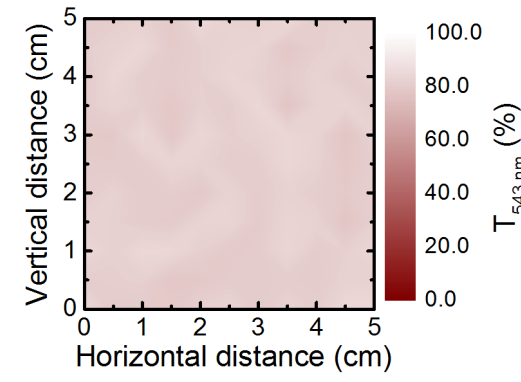

(d)
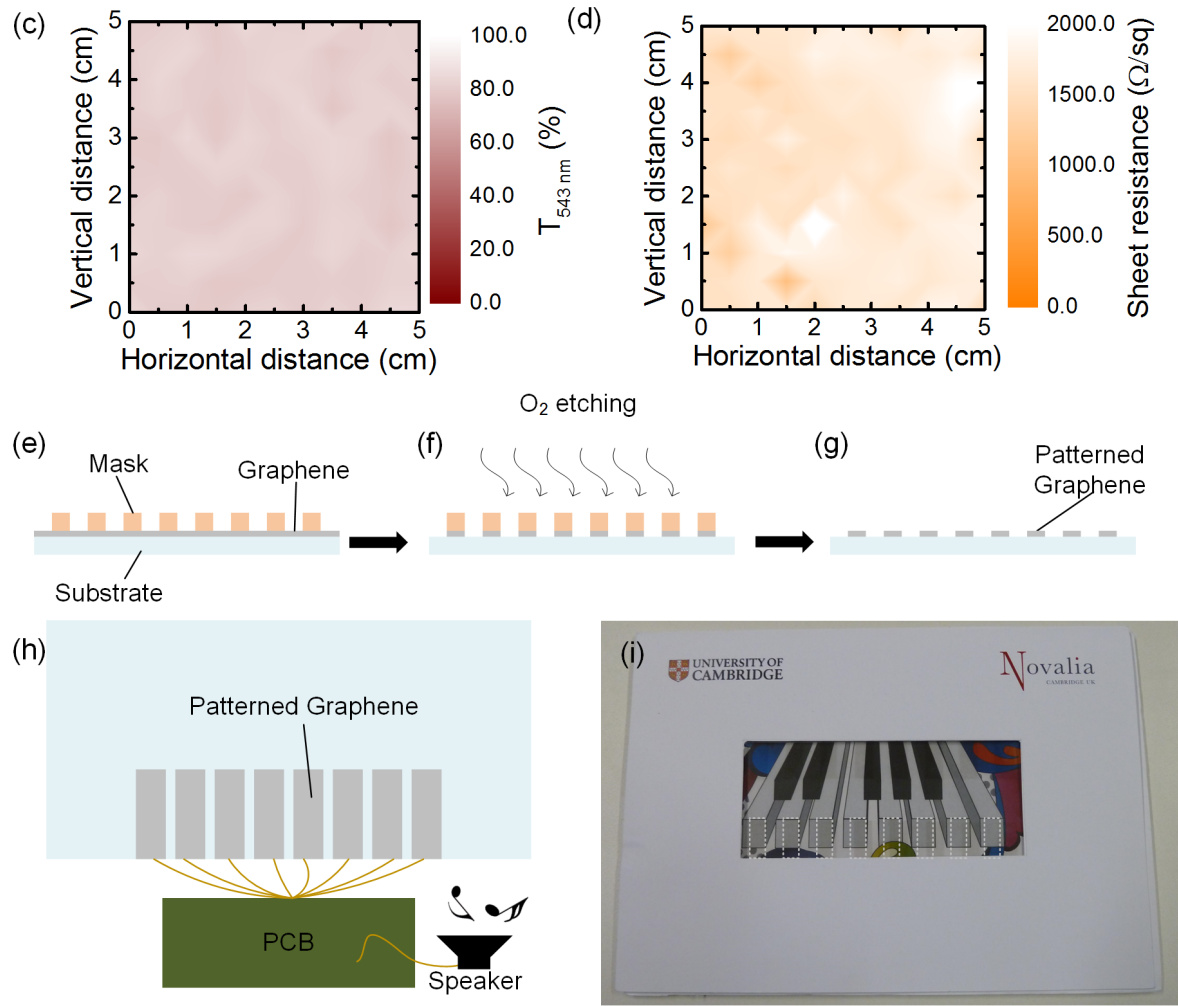

Figure 3. a) Graphene-based TCE (outlined in red). b) Optical transmittance of the film, showing 80\% for the coated substrate across the visible spectrum. c) Optical and d) electrical uniformity of the film. e-g) Patterning steps of the TCE via oxygen plasma etching. h, i) Flexible transparent piano assembled using the electrode after patterning, with white lines indicating the edge of the "keys". 
One of the key potential applications for solution processed and CVD graphene is as transparent conducting electrodes (TCE).1,12,83-85 While CVD graphene produces a single continuous layer of material, enabling high electrical conductivity ${ }^{12,43}$ and significant progress has been made for roll-to-roll graphene growth, ${ }^{12,39}$ considerations on costs, scalability and device dimensions favor the use of solution processed graphene for applications requiring moderate sheet resistance. ${ }^{1,83,84,86}$ Solution processing allows uniform films to be deposited across large areas, vital for consistent device manufacture and performance. In solution-based graphene TCEs, the main source of resistance is the interfaces between flakes. ${ }^{1,84,87}$ Hybrid films containing graphene along with $1 \mathrm{~d}$ materials such as carbon nanotubes ${ }^{88,89}$ can show improved performance by increasing the number of conductive pathways through the TCE film.

We have used a graphene-based ink formulated for Mayer-bar printing to deposit a flexible TCE on polyethylene terephthalate (PET) substrate (Fig. 3a). Mayer bars consist of a central rod wound with a tight coil of wire, and were first developed around 100 years ago to meter carbon and wax paper coatings. ${ }^{90}$ They are now widely used to deposit uniform thickness films on flexible substrates in roll-to-roll systems. ${ }^{90}$ The ink we have formulated has a low surface tension, allowing it to readily wet the substrate prior to solvent evaporation, ensuring a consistent coating. The fabricated electrode has good electrical and optical uniformity, (Fig. 3c, d). The optical transmittance across the visible spectrum is $\sim 80 \%$ including the substrate, or $>90 \%$ for the coating alone (Fig. $3 \mathrm{~b}$ ), while the transmission at $543 \mathrm{~nm}$ has $<5 \%$ standard deviation across several $\mathrm{cm}^{2}$ of the electrode (Fig. 3c). The average sheet resistance is $\sim 900 \Omega /$ sq, again with $<5 \%$ standard deviation (Fig. 3d). The TCE can be patterned by masking followed by $\mathrm{O}_{2}$ plasma etching (Fig. 3e-g).

Such electrode arrays can then be interfaced with traditional electronics circuitry. An example of this is shown (Fig. 3h, i), where a patterned TCE is used to build a capacitive touchpad piano working in collaboration with Novalia Ltd. The TCE is interfaced with a PCB using copper tracks, while the individual "touchpads" are covered with a thin polymer layer acting as the dielectric. The PCB can then detect the changes in capacitance when one of the "keys" is touched. When this occurs, the electronics on the PCB process the signal and play the appropriate sound through an integrated, thin speaker module (Fig. 3h, i). This demonstrates how advanced nanomaterials could be directly integrated with existing electronics circuitry, bringing together inexpensive, widely available and programmable PCB circuitry with the flexible form factor of printed/coated graphene-based TCEs.

\subsection{Spray-coated graphene for electroluminescent devices}
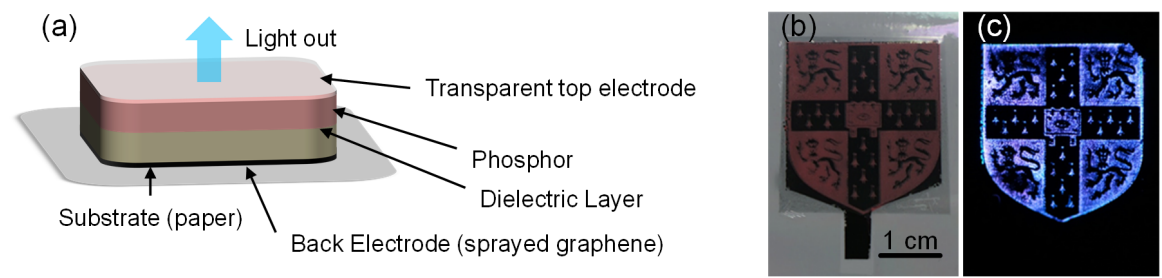

Figure 4. Electroluminescent display with spray-coated graphene electrode. a) schematic of the device structure. The display in the b) OFF and c) ON states.

For certain device applications, the priority is low cost and high conductivity on flexible substrates (rather than transparency). Through UALPE of graphene in isopropanol (IPA), we are able to produce a high concentration $\left(\sim 1 \mathrm{gL}^{-1}\right)$ graphene ink suitable for spray-coating. While the stability of the ink is only several hours, it can be uniformly spray-coated on to the target substrates. The low boiling-point of the carrier solvent means that the ink drying time is very short and electrodes can be rapidly deposited over a large area. The uniformity of the sprayed graphene can be further improved by corona (air plasma) or oxygen plasma treatment of the substrate prior to spraying. The electrodes have a sheet resistance of $\sim 250 \Omega / \mathrm{sq}$, with good consistency across the device area ( $\sim 5 \%$ standard deviation).

We have used these spray-coated electrodes as the back-plane for an electroluminescent (EL) device. EL devices are commonly used for applications such as backlighting, as they offer efficient and uniform light generation across a large area. The device consists of two electrodes sandwiching a layer of phosphor and a dielectric 
(Fig. 4a). When an alternating electric field is applied across the phosphor, light is produced. In our device, the graphene is spray-coated onto a paper substrate, followed by printing of a commercial phosphor (DuPont LuxPrint $8150 \mathrm{~L}$ ). We use a $\sim 1.5 \mu \mathrm{m}$ thick laminated polymer film as the dielectric. This allows a simple and pinhole free dielectric layer while offering a significant thickness and cost reduction over conventional printed dielectric layers (typically $\sim 60 \mu \mathrm{m}$ thick, $\sim 1500 \mathrm{USD} / \mathrm{kg}$ ). A transparent electrode consisting of spray-coated PEDOT:PSS completes the device. Excluding the paper substrate, the overall device thickness is $\sim 40 \mu \mathrm{m}$, with the majority of this $(\sim 35 \mu \mathrm{m})$ being due to the phosphor. Fig. $4 \mathrm{~b}, \mathrm{c}$ shows the device in both OFF and ON states, demonstrating the suitability of our electrode for conformable and wearable EL devices.

\subsection{Inkjet printed $2 \mathrm{~d}$ materials for electronics and photonics}
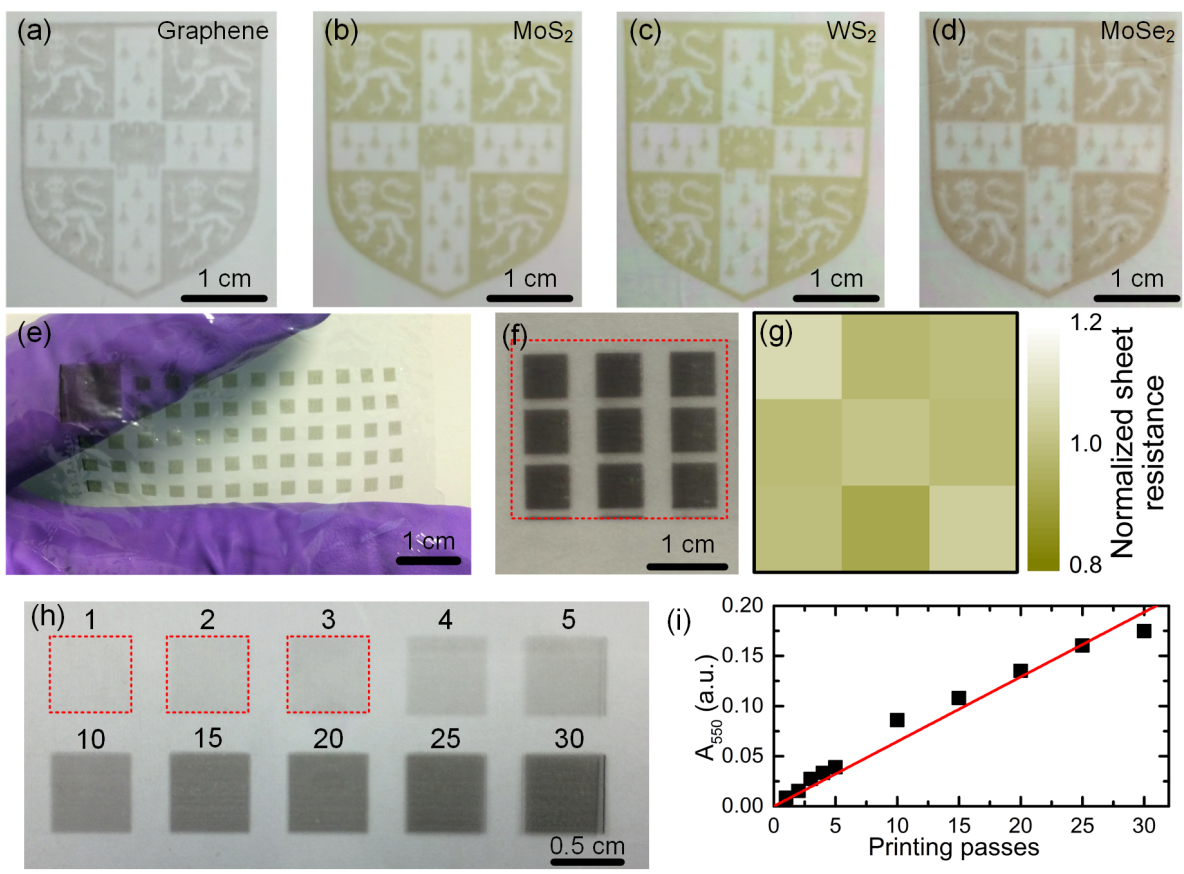

Figure 5. Inkjet printed 2d materials. a) Graphene, b) $\mathrm{MoS}_{2}$, c) $\mathrm{WS}_{2}$, and d) $\mathrm{MoSe}_{2}$ printed on PET. Arrays of graphene squares printed on e) LDPE, f) glass. g) Normalized sheet resistance of printed graphene shown in $\mathrm{f}$ ). h) Array of graphene squares of different printing passes on glass. i) optical absorption of the printed squares shown in h) at $550 \mathrm{~nm}$.

Inkjet is a digital printing technique, widely used for high resolution $(10-100 \mu \mathrm{m})$ patterning of substrates. ${ }^{91}$ Droplets are produced as required by the pattern, and generated by a piezoelectric or heating element driving ink through a nozzle. This maskless, additive deposition process not only allows high precision $(\sim 20 \mu \mathrm{m})$ in position also in terms of ink volume $(\sim 10 \mathrm{pL} /$ droplet $){ }^{91}$ We have recently developed a series of graphene and s-TMD based inkjet printable inks.

For reliable inkjet printing, it is necessary to achieve stable single droplet jetting (i.e. one droplet for each printer input, with no satellite droplet formation). A commonly used figure of merit for ink jetting is Z:

$$
Z=\frac{(\gamma \rho a)^{\frac{1}{2}}}{\eta}
$$

where $\gamma$ is the surface tension of the ink $\left(\mathrm{mNm}^{-1}\right), \rho$ is the density of the ink $\left(\mathrm{gcm}^{-3}\right), \eta$ is the viscosity of the ink (mPa.s) and $a$ is the nozzle diameter $(\mu \mathrm{m}) .{ }^{91-94}$ As a rule of thumb, it is commonly accepted that $1<\mathrm{Z}<14$ is required for stable drop-on-demand inkjet printing. ${ }^{91-93}$ Inks with $\mathrm{Z}<1$ dissipate the jetting actuation from the printer too rapidly to form a droplet, while those with $\mathrm{Z}>14$ produce additional satellite droplets. ${ }^{91-93}$ It should 
be noted, however, that while $\mathrm{Z}$ acts as a useful guide, inks falling outside this range may also be printable, for example by controlling the frequency and amplitude of the jetting impulses..$^{91,95}$

The graphene ink is produced via UALPE of graphite in IPA, but differs from the spray-coating ink discussed in section 4.2. As discussed earlier, graphene dispersions in pure IPA are metastable due to the Hansen parameter mismatch, rendering them unsuitable for inkjet printing. ${ }^{53,74}$ We therefore use polyvinylpyrollidone (PVP, 0.15 $\mathrm{gL}^{-1}$ ) as a stabilizer for the exfoliated graphene flakes, as previously demonstrated with carbon nanotubes. ${ }^{96} \mathrm{In}$ addition to aiding the stability of the graphene ink, PVP also modifies the rheology of the ink to improve its printability. We measure $\gamma$ and $\eta$ for the graphene-PVP ink, via a pendant droplet method and parallel plate rheometer respectively, and working at room temperature. We determine $\gamma \sim 28 \mathrm{mNm}^{-1}$ and $\eta \sim 2.3 \mathrm{mPas}$. On the Dimatix DMP-2381 used for this work, $a=22 \mu \mathrm{m}$. With $\rho \sim 0.8 \mathrm{gcm}^{-3}$, this gives $\mathrm{Z} \sim 9.6$, falling within the recommended range for stable jetting.

The s-TMD inks, meanwhile, are formulated from dispersions produced via UALPE of their bulk crystals in cyclohexanone. This allows exfoliation without the need for dispersants. As with the graphene ink above, we can determine $\mathrm{Z}$ for the inks to ensure that they will be printable. Using the same methods as before, we measure $\gamma \sim 34 \mathrm{mNm}^{-1}, \eta \sim 2 \mathrm{mPas}$, and $\rho \sim 1.0 \mathrm{gcm}^{-3}$. This gives $\mathrm{Z} \sim 13.4$, again indicating the inkjet printability of the formulation.

The inks can be printed on to a variety of substrates, including glass, as well as flexible and conformable substrates such as PET and low density polyethylene (LDPE), some examples of which are shown in Fig. 5. High resolution and high uniformity printing is possible across all of these substrates (Fig. 5a-f, h).

We can confirm the uniformity of the printed graphene by measuring the sheet resistance of an array of identical printed squares. For this, 9 squares are printed on a glass substrate (Fig. 5f). After annealing to remove the residual PVP, the variation in sheet resistance across the patterns is measured (Fig. 5d). As can be seen, the pattern is very uniform, with $<5 \%$ standard deviation in the sheet resistance between the 9 squares.

In addition to precise spatial positioning, inkjet printing allows good control over the volume of ink deposited, which allows tailoring of the printed structures to the desired application. With a view to photonics applications, discussed further in section 4.4, we have printed squares with different numbers of printing passes (Fig. 5h), allowing the absorption to be varied. The optical absorption and printing pass number are well correlated, with $<5 \%$ standard deviation (Fig. 5h, i). Gaining precise control over optical absorption is critical for photonic applications. For example, in the case of saturable absorbers (discussed in section 4.4), control over absorbance allows simplified design of laser cavities for ultrafast pulse generation. ${ }^{97}$

\subsection{Photonic composites for ultrafast pulsed lasers}

Moving beyond applications in electronics and optoelectronics, 2d material inks and dispersions are of strong interest in the field of photonics, in particular, for laser sources producing nanosecond to sub-picosecond optical pulses. Such ultrafast laser systems are a major component in the portfolio of leading laser manufacturers and are of high demand in a wide variety of applications ranging from basic scientific research to materials processing, and from eye surgery, spectroscopy and biomedical imaging to printed circuit-board manufacturing. ${ }^{98-101}$ The majority of the ultrafast laser systems exploit nonlinear optical materials or devices, commonly known as saturable absorbers (SAs), which exhibit reduced optical absorption under intense illumination. ${ }^{100,102}$ These can be used to generate ultrafast pulses, either through mode-locking ${ }^{99,103}$ or Q-switching. ${ }^{98,103}$

In a mode-locked laser, the SA is used to couple the longitudinal modes that constitute a laser output. ${ }^{103}$ Points in the waveform where these modes are in phase (high intensity) are selectively amplified, while points where they are in antiphase (low intensity) are damped. ${ }^{103}$ This produces a train of high repetition rate $(\mathrm{MHz}-$ $\mathrm{GHz}$ ) and short (fs-ps) pulses. ${ }^{99,103}$ In a Q-switched laser, the SA modulates the Q-factor (i.e. the resonance damping) of the laser cavity. ${ }^{103}$ The unsaturated SA gives a low Q-factor, causing energy to build up in the cavity. When sufficient energy builds up, the SA saturates, causing a rapid increase in the cavity Q-factor, leading to a high energy pulse output. ${ }^{103}$ This reduces the energy in the cavity, returning to the unsaturated condition. Q-switched lasers typically produce relatively long (ns- $\mu \mathrm{s})$ and high energy $(\mu \mathrm{J}-\mathrm{mJ})$ pulses at lower repetition rates than mode-locked lasers (typically $\mathrm{kHz}$ ). ${ }^{98,103}$ 
(a)

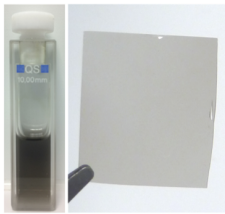

(b)

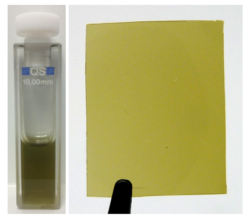

(c)

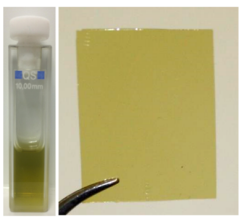

(d)

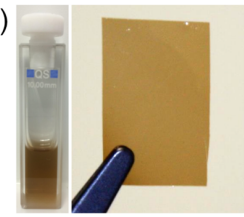

(e)

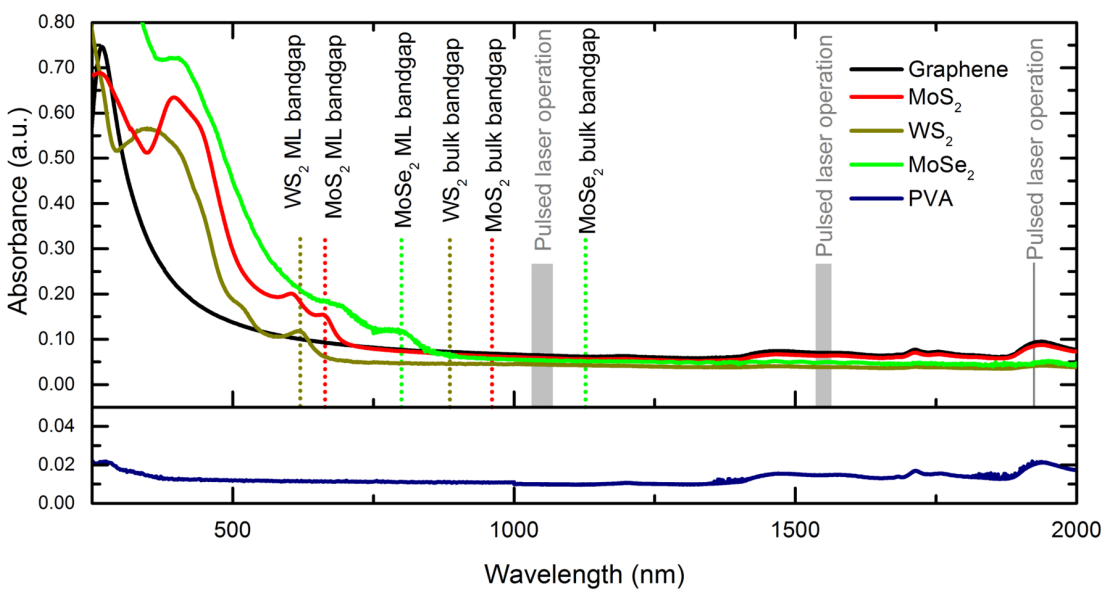

(f)

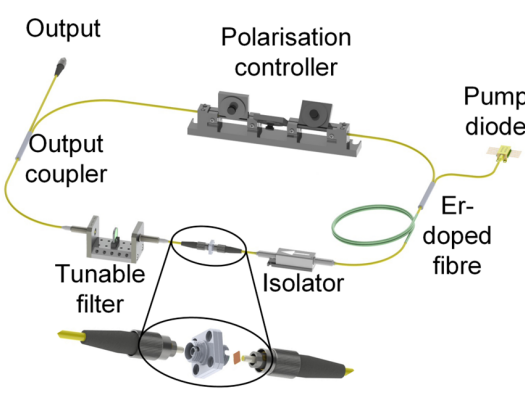

SA film between fibre patchcords

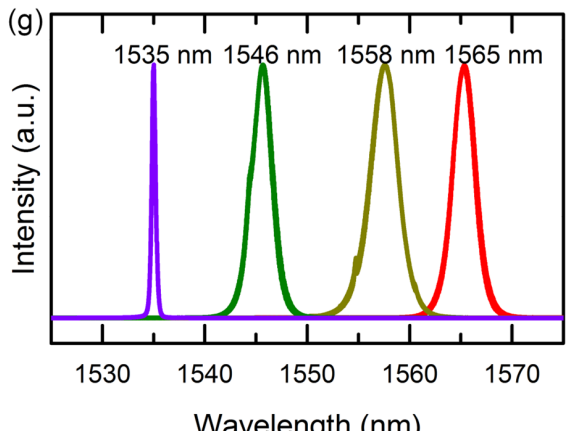

Figure 6. s-TMD dispersions and composites for ultrafast pulsed lasers. Dispersions and free-standing polymer composite films of a) graphene, b) $\mathrm{MoS}_{2}$, c) $\mathrm{WS}_{2}$, and d) $\mathrm{MoSe}_{2}$. e) optical absorbance of the composite films. The absorbance of an equivalent PVA film is shown for reference. f) integration into a fiber laser cavity for tunable mode-locking. g) example tunable pulsed output from a $\mathrm{MoS}_{2}$-based mode-locked laser.

The currently dominant SA technology is semiconductor saturable absorber mirror (SESAM). ${ }^{102}$ However, limitations such as the fabrication complexity, cost and relatively narrowband tunability have driven research into alternatives, with $1 \mathrm{~d}$ and $2 \mathrm{~d}$ materials emerging as promising candidates. Among the $2 \mathrm{~d}$ materials, it has been demonstrated that graphene, ${ }^{104,105}$ s-TMDs $^{106}$ and $\mathrm{BP}^{30,107,108}$ display ultrafast carrier dynamics and nonlinear saturable absorption, making them suitable for ultrafast pulse generation.

In particular, graphene, with its linear dispersion of Dirac electrons, displays broadband saturable absorption across the visible and near-infrared spectrum. ${ }^{101,104}$ Meanwhile, s-TMDs have significant potential for visiblewavelength SA operation due to their direct bandgaps. They additionally exhibit linear absorption below the material bandgap. Along with several other groups, ${ }^{109-111}$ we have experimentally shown that this absorption can be saturated under high irradiation. ${ }^{57,59,60,82,112}$ Absorption measurements using an integrating sphere show that $\sim 10 \%$ of the measured absorption from the $2 \mathrm{~d}$ material flakes is due to scattering. We have attributed the remaining $\sim 90 \%$ to edge-mediated states resulting from the high edge to surface area ratio of the exfoliated nanoflakes. ${ }^{57,59,60,82,112}$ This is consistent with the previous experiments, which showed an increase in sub-bandgap absorption for lithographically patterned $\mathrm{MoS}_{2}$ crystals. ${ }^{113}$ This sub-bandgap absorption allows s-TMDs, much 
like graphene, to be used as SAs across a broad range of wavelengths, in the near infrared and potentially in the visible range. Thus, these layered materials, in addition to BP, have recently received tremendous interest as low cost SAs for ultrafast pulse generation (graphene, ${ }^{56,100,104}$ s-TMDs, ${ }^{57-60,106,112,114} \mathrm{BP}^{107,108}$ ).

Solution processed $2 \mathrm{~d}$ materials offers additional benefits, allowing them to be readily integrated into laser cavities, either via printing and coating onto optical components such as quartz substrates, fiber facets and mirrors, or through blending with host polymers to produce composite films. ${ }^{100,101,112,115}$ In particular, for ease of handling and integration, free-standing polymer-composites are highly preferable. ${ }^{100,116}$ We have taken this approach to produce SA films using graphene, as well as a variety of s-TMDs $\left(\mathrm{MoS}_{2}, \mathrm{MoSe}_{2}, \mathrm{WS}_{2}\right)$. The dispersions are produced by UALPE in water, as this allows the composites to be prepared at room temperature. As with IPA, the large Hansen parameter mismatch between water and these $2 \mathrm{~d}$ materials necessitates the use of a dispersant (such as a surfactant) to support a stable dispersion. ${ }^{53}$ We have shown that the most effective surfactants for $2 \mathrm{~d}$ materials are those with a quasi-2d structure with hydrophilic and hydrophobic faces (e.g. bile salts). ${ }^{56,97}$ The hydrophobic face of the molecule adsorbs onto the $2 \mathrm{~d}$ surface, encapsulating exfoliated flakes and stabilizing the dispersion. ${ }^{53,56}$ Using such a bile salt surfactant, sodium deoxycholate (SDC), we get (on average) 60-80 nm flakes with 4-5 nm thickness (5-7 layers) with an estimated material concentration of $\sim 0.6-\sim 0.9 \mathrm{gL}^{-1} .{ }^{57,59,60,82,97}$ This gives an edge to surface area ratio ranging from 1:6 to 1:10 for our UALPE flakes. A typical example of the stable dispersions is shown in Fig. 6a-d.

\begin{tabular}{|c|c|c|c|c|}
\hline Gain-band & Graphene & $\mathrm{MoS}_{2}$ & $\mathrm{WS}_{2}$ & $\mathrm{MoSe}_{2}$ \\
\hline Ytterbium & - & $\begin{array}{c}\text { QS }-1068 \mathrm{~nm}^{58} \\
\text { QS - 1030-1070 nm tunable }\end{array}$ & QS - $1030 \mathrm{~nm}^{82,117}$ & QS - $1060 \mathrm{~nm}^{60}$ \\
\hline Erbium & $\begin{array}{c}\text { ML - } 1525-1559 \mathrm{~nm} \text { tunable } \\
\text { ML }-1562 \mathrm{~nm}^{118} \\
\text { ML - } 1559 \mathrm{~nm}^{104,119} \\
\text { QS - } 1522-1555 \mathrm{~nm} \text { tunable } \\
120\end{array}$ & ML - $1535-1565 \mathrm{~nm}$ tunable ${ }^{57}$ & QS - $1560 \mathrm{~nm}^{82}$ & QS - $1560 \mathrm{~nm}^{60}$ \\
\hline Thulium & ML - $1940 \mathrm{~nm}^{121}$ & - & - & QS - $1924 \mathrm{~nm}^{60}$ \\
\hline
\end{tabular}

Table 1. Summary of ultrafast lasers mode-locked (ML) and Q-switched (QS) by our 2d material composite films.

The composite films are fabricated by blending these dispersions with an aqueous solution of polyvinyl alcohol (PVA); Fig. 6a-d. Slow evaporation of water at room temperature produces $\sim 25-30 \mu$ m thick freestanding polymer composite SAs. The SAs can be integrated into the fiber laser cavity by sandwiching $\sim 2 \mathrm{~mm}^{2}$ composites between two fiber ends (Fig. 6f). The first demonstration of graphene mode locked laser exploited such solution processing strategy. ${ }^{100,104}$ We used a similar strategy to generate ultrafast pulses using s-TMD based SAs. An example output from a mode-locked laser using a $\mathrm{MoS}_{2} \mathrm{SA}$ is shown in Fig. 6g. This shows consistent pulse output across the tuning range of $1535-1565 \mathrm{~nm}$. We note that the range of tuning is limited by that of the tunable filter, rather than the $\mathrm{MoS}_{2} \mathrm{SA}$. The pulses have a typical duration of $\sim 1 \mathrm{ps,}$ energy of $\sim 65 \mathrm{pJ}$, and repetition rate of $\sim 13 \mathrm{MHz}$. Overall, we have demonstrated graphene and s-TMD-polymer composite SAs to mode-lock and Q-switch ytterbium- $(\sim 1030 \mathrm{~nm})$, erbium- $(\sim 1550 \mathrm{~nm})$ and thulium-doped $(\sim 1930 \mathrm{~nm})$ fiber lasers. Selected results, presented in Table 1 demonstrate the suitability of both graphene ${ }^{56,104,118-121}$ and sTMDs $^{57-60,82,117}$ as SAs in the 1-2 $\mu \mathrm{m}$ range. We note that while gaps exist in this table, they are filled by results from other research groups, a summary of which is beyond the scope of this manuscript. ${ }^{112,116}$

\section{CONCLUSIONS}

We have demonstrated a range of (opto)electronic devices using functional inks of $2 \mathrm{~d}$ materials. As the library of $2 \mathrm{~d}$ materials grows, more exotic materials and properties are likely to emerge, in particular when different materials are combined to produce heterostructures. Indeed, such heterostructures could exceed the (opto)electronic properties of individual materials and significantly improve the application scope of these materials. As a low cost and adaptable platform, we foresee that solution processing and printing will play a key role in the commercialization and exploitation of $2 \mathrm{~d}$ materials and their heterostructures in the near future. 


\section{ACKNOWLEDGMENTS}

The authors would like to acknowledge E.J.R. Kelleher, R.I. Woodward, M. Zhang, J.R. Taylor, Z. Sun, S. Schofield, Y. Li, C. Jones, K. Stone, S. Jones and N. Chilton for useful discussions in the preparation of this manuscript. We acknowledge funding from EPSRC grants EP/G037221/1 and EP/K503757/1 (Graflex) and a research fellowship from the Royal Academy of Engineering.

Data used in this publication can be accessed at: https://www.repository.cam.ac.uk/handle/1810/249246

\section{REFERENCES}

[1] Bonaccorso, F., Sun, Z., Hasan, T., and Ferrari, A. C., "Graphene photonics and optoelectronics," Nat. Photonics 4(9), 611-622 (2010).

[2] Wang, Q. H., Kalantar-Zadeh, K., Kis, A., Coleman, J. N., and Strano, M. S., "Electronics and optoelectronics of two-dimensional transition metal dichalcogenides," Nat. Nanotechnol. 7(11), 699-712 (2012).

[3] Xu, M., Liang, T., Shi, M., and Chen, H., "Graphene-like two-dimensional materials," Chem. Rev. 113(5), 3766-98 (2013).

[4] Liu, H., Du, Y., Deng, Y., and Ye, P. D., "Semiconducting black phosphorus: synthesis, transport properties and electronic applications," Chem. Soc. Rev. 44(9), 2732-2743 (2015).

[5] Castro Neto, A. H., Guinea, F., Peres, N. M. R., Novoselov, K. S., and Geim, A. K., "The electronic properties of graphene," Rev. Mod. Phys. 81(1), 109-162 (2009).

[6] Geim, A. K. and Novoselov, K. S., "The rise of graphene," Nat. Mater. 6(3), 183-191 (2007).

[7] Novoselov, K. S., Geim, A. K., Morozov, S. V., Jiang, D., Zhang, Y., Dubonos, S. V., Grigorieva, I. V., and Firsov, A. A., "Electric field effect in atomically thin carbon films," Science 306(5696), 666-9 (2004).

[8] Nair, R. R., Blake, P., Grigorenko, A. N., Novoselov, K. S., Booth, T. J., Stauber, T., Peres, N. M. R., and Geim, A. K., "Fine structure constant defines visual transparency of graphene," Science 320(5881), 1308 (2008).

[9] Mak, K. F., Ju, L., Wang, F., and Heinz, T. F., "Optical spectroscopy of graphene: From the far infrared to the ultraviolet," Solid State Commun. 152(15), 1341-1349 (2012).

[10] Kravets, V. G., Grigorenko, A. N., Nair, R. R., Blake, P., Anissimova, S., Novoselov, K. S., and Geim, A. K., "Spectroscopic ellipsometry of graphene and an exciton-shifted van Hove peak in absorption," Phys. Rev. B 81(15), 1-6 (2010).

[11] Lee, C., Wei, X., Kysar, J. W., and Hone, J., "Measurement of the elastic properties and intrinsic strength of monolayer graphene," Science 321(5887), 385-8 (2008).

[12] Bae, S., Kim, H., Lee, Y., Xu, X., Park, J.-S., Zheng, Y., Balakrishnan, J., Lei, T., Kim, H. R., Song, Y. I., Kim, Y.-J., Kim, K. S., Ozyilmaz, B., Ahn, J.-H., Hong, B. H., and Iijima, S., "Roll-to-roll production of 30-inch graphene films for transparent electrodes," Nat. Nanotechnol. 5(8), 574-578 (2010).

[13] Kam, K. K. and Parkinson, B. A., "Detailed photocurrent spectroscopy of the semiconducting group VI transition metal dichalcogenides," J. Phys. Chem. 86(4), 463-467 (1982).

[14] Schwierz, F., "Graphene transistors," Nat. Nanotechnol. 5(7), 487-496 (2010).

[15] Radisavljevic, B., Radenovic, A., Brivio, J., Giacometti, V., and Kis, A., "Single-layer $\mathrm{MoS}_{2}$ transistors," Nat. Nanotechnol. 6(3), 147-150 (2011).

[16] Radisavljevic, B., Whitwick, M. B., and Kis, A., "Integrated circuits and logic operations based on singlelayer $\mathrm{MoS}_{2}, "$ ACS Nano 5(12), 9934-8 (2011).

[17] Fuhrer, M. S. and Hone, J., "Measurement of mobility in dual-gated $\mathrm{MoS}_{2}$ transistors," Nat. Nanotechnol. 8(3), 146-7 (2013).

[18] Radisavljevic, B., Whitwick, M. B., and Kis, A., "Small-signal amplifier based on single-layer $\mathrm{MoS}_{2}$," Appl. Phys. Lett. 101(4), 043103 (2012).

[19] Wang, H., Yu, L., Lee, Y.-H., Shi, Y., Hsu, A., Chin, M. L., Li, L.-J., Dubey, M., Kong, J., and Palacios, T., "Integrated circuits based on bilayer $\mathrm{MoS}_{2}$ transistors," Nano Lett. 12(9), 4674-4680 (2012).

[20] Radisavljevic, B. and Kis, A., "Reply to "Measurement of mobility in dual-gated $\mathrm{MoS}_{2}$ transistors'," Nat. Nanotechnol. 8(3), 147-8 (2013). 
[21] Mak, K. F., Lee, C., Hone, J., Shan, J., and Heinz, T. F., "Atomically thin $\mathrm{MoS}_{2}$ : A new direct-gap semiconductor," Phys. Rev. Lett. 105(13), 136805 (2010).

[22] Splendiani, A., Sun, L., Zhang, Y., Li, T., Kim, J., Chim, C.-Y., Galli, G., and Wang, F., "Emerging photoluminescence in monolayer $\mathrm{MoS}_{2}, "$ Nano Lett. 10(4), 1271-5 (2010).

[23] Sundaram, R. S., Engel, M., Lombardo, A., Krupke, R., Ferrari, A. C., Avouris, P., and Steiner, M., "Electroluminescence in single layer $\mathrm{MoS}_{2}, "$ Nano Lett. 13(4), 1416-21 (2013).

[24] Lee, H. S., Min, S.-W., Chang, Y.-G., Park, M. K., Nam, T., Kim, H., Kim, J. H., Ryu, S., and Im, S., "MoS $\mathrm{M}_{2}$ nanosheet phototransistors with thickness-modulated optical energy gap," Nano Lett. 12(7), 3695-3700 (2012).

[25] Yin, Z., Li, H., Li, H., Jiang, L., Shi, Y., Sun, Y., Lu, G., Zhang, Q., Chen, X., and Zhang, H., "Single-layer $\mathrm{MoS}_{2}$ phototransistors," ACS Nano 6(1), 74-80 (2012).

[26] Finn, D. J., Lotya, M., Cunningham, G., Smith, R. J., McCloskey, D., Donegan, J. F., and Coleman, J. N., "Inkjet deposition of liquid-exfoliated graphene and $\mathrm{MoS}_{2}$ nanosheets for printed device applications," $J$. Mater. Chem. C 2(5), 925-932 (2014).

[27] Frindt, R. F. and Yoffe, A. D., "Physical properties of layer structures: Optical properties and photoconductivity of thin crystals of molybdenum disulphide," Proc. R. Soc. A 273(1352), 69-83 (1963).

[28] Han, C. Q., Yao, M. Y., Bai, X. X., Miao, L., Zhu, F., Guan, D. D., Wang, S., Gao, C. L., Liu, C., Qian, D., Liu, Y., and Jia, J. F., "Electronic structure of black phosphorus studied by angle-resolved photoemission spectroscopy," Phys. Rev. B 90(8), 2-6 (2014).

[29] Zhang, S., Yang, J., Xu, R., Wang, F., Li, W., Ghufran, M., Zhang, Y.-W., Yu, Z., Zhang, G., Qin, Q., and $\mathrm{Lu}$, Y., "Extraordinary photoluminescence and strong temperature/angle-dependent Raman responses in few-layer phosphorene," ACS Nano 8(9), 9590-9596 (2014).

[30] Hanlon, D., Backes, C., Doherty, E., Cucinotta, C. S., Berner, N. C., Boland, C., Lee, K., Lynch, P., Gholamvand, Z., Harvey, A., Zhang, S., Wang, K., Moynihan, G., Pokle, A., Ramasse, Q. M., McEvoy, N., Blau, W. J., Wang, J., Sanvito, S., O'Regan, D. D., Duesberg, G. S., Nicolosi, V., and Coleman, J. N., "Liquid exfoliation of solvent-stabilised black phosphorus: applications beyond electronics," arXiv $\mathbf{1 5 0 1 . 0 1 8 8 1}$ (2015).

[31] Li, L., Yu, Y., Ye, G. J., Ge, Q., Ou, X., Wu, H., Feng, D., Chen, X. H., and Zhang, Y., "Black phosphorus field-effect transistors," Nat. Nanotechnol. 9(5), 372-7 (2014).

[32] Castellanos-Gomez, A., Vicarelli, L., Prada, E., Island, J. O., Narasimha-Acharya, K. L., Blanter, S. I., Groenendijk, D. J., Buscema, M., Steele, G. A., Alvarez, J. V., Zandbergen, H. W., Palacios, J. J., and van der Zant, H. S. J., "Isolation and characterization of few-layer black phosphorus," 2D Mater. 1(2), 025001 (2014).

[33] Wood, J. D., Wells, S. A., Jariwala, D., Chen, K.-S., Cho, E., Sangwan, V. K., Liu, X., Lauhon, L. J., Marks, T. J., and Hersam, M. C., "Effective passivation of exfoliated black phosphorus transistors against ambient degradation," Nano Lett. 14(12), 6964-6970 (2014).

[34] Engel, M., Steiner, M., and Avouris, P., "Black phosphorus photodetector for multispectral, high-resolution imaging," Nano Lett. 14(11), 6414-6417 (2014).

[35] Hasan, M. Z. and Kane, C. L., "Topological insulators," Rev. Mod. Phys. 82(4), 3045-3067 (2010).

[36] Naguib, M., Come, J., Dyatkin, B., Presser, V., Taberna, P.-l., Simon, P., Barsoum, M. W., and Gogotsi, Y., "MXene: a promising transition metal carbide anode for lithium-ion batteries," Electrochem. Commun. 16(1), 61-64 (2012).

[37] Naguib, M., Mochalin, V. N., Barsoum, M. W., and Gogotsi, Y., "MXenes: A new family of twodimensional materials," Adv. Mater. 26(7), 992-1005 (2014).

[38] Annabi-Bergaya, F., "Layered clay minerals. Basic research and innovative composite applications," Microporous Mesoporous Mater. 107(1), 141-148 (2008).

[39] Bonaccorso, F., Lombardo, A., Hasan, T., Sun, Z., Colombo, L., and Ferrari, A. C., "Production and processing of graphene and 2d crystals," Mater. Today 15(12), 564-589 (2012).

[40] Lee, H. S., Min, S.-W., Park, M. K., Lee, Y. T., Jeon, P. J., Kim, J. H., Ryu, S., and Im, S., "MoS nanosheets for top-gate nonvolatile memory transistor channel," Small 8(20), 3111-5 (2012). 
[41] Liu, K.-K., Zhang, W., Lee, Y.-H., Lin, Y.-C., Chang, M.-T., Su, C.-Y., Chang, C.-S., Li, H., Shi, Y., Zhang, H., Lai, C.-S., and Li, L.-J., "Growth of large-area and highly crystalline $\mathrm{MoS}_{2}$ thin layers on insulating substrates," Nano Lett. 12(3), 1538-44 (2012).

[42] Li, X., Cai, W., An, J., Kim, S., Nah, J., Yang, D., Piner, R., Velamakanni, A., Jung, I., Tutuc, E., Banerjee, S. K., Colombo, L., and Ruoff, R. S., "Large-area synthesis of high-quality and uniform graphene films on copper foils," Science 324(5932), 1312-4 (2009).

[43] Kim, K. S., Zhao, Y., Jang, H., Lee, S. Y., Kim, J. M., Kim, K. S., Ahn, J.-H., Kim, P., Choi, J.-Y., and Hong, B. H., "Large-scale pattern growth of graphene films for stretchable transparent electrodes," Nature 457(7230), 706-710 (2009).

[44] Lee, Y.-H., Yu, L., Wang, H., Fang, W., Ling, X., Shi, Y., Lin, C.-T., Huang, J.-K., Chang, M.-T., Chang, C.-S., Dresselhaus, M., Palacios, T., Li, L.-J., and Kong, J., "Synthesis and transfer of single-layer transition metal disulfides on diverse surfaces," Nano Lett. 13(4), 1852-7 (2013).

[45] Gurarslan, A., Yu, Y., Su, L., Yu, Y., Suarez, F., Yao, S., and Zhu, Y., "Surface-energy assisted perfect transfer of centimeter-scale films onto arbitrary substrates," ACS Nano 8(11), 11522-11528 (2014).

[46] Reina, A., Jia, X., Ho, J., Nezich, D., Son, H., Bulovic, V., Dresselhaus, M. S., and Kong, J., "Large area, few-layer graphene films on arbitrary substrates by chemical vapor deposition," Nano Lett. 9(1), 30-35 (2009).

[47] Li, X., Zhu, Y., Cai, W., Borysiak, M., Han, B., Chen, D., Piner, R. D., Colomba, L., and Ruoff, R. S., "Transfer of large-area graphene films for high-performance transparent conductive electrodes," Nano Lett. 9(12), 4359-4363 (2009).

[48] Najmaei, S., Liu, Z., Zhou, W., Zou, X., Shi, G., Lei, S., Yakobson, B. I., Idrobo, J.-C., Ajayan, P. M., and Lou, J., "Vapour phase growth and grain boundary structure of molybdenum disulphide atomic layers.," Nat. Mater. 12(8), 754-9 (2013).

[49] Pirkle, A., Chan, J., Venugopal, A., Hinojos, D., Magnuson, C. W., McDonnell, S., Colombo, L., Vogel, E. M., Ruoff, R. S., and Wallace, R. M., "The effect of chemical residues on the physical and electrical properties of chemical vapor deposited graphene transferred to $\mathrm{SiO}_{2}, "$ Appl. Phys. Lett. 99(12), 122108 (2011).

[50] Chan, J., Venugopal, A., Pirkle, A., McDonnell, S., Hinojos, D., Magnuson, C. W., Ruoff, R. S., Colombo, L., Wallace, R. M., and Vogel, E. M., "Reducing extrinsic performance-limiting factors in graphene grown by chemical vapor deposition," ACS Nano 6(4), 3224-3229 (2012).

[51] Coleman, J. N., Lotya, M., O’Neill, A., Bergin, S. D., King, P. J., Khan, U., Young, K., Gaucher, A., De, S., Smith, R. J., Shvets, I. V., Arora, S. K., Stanton, G., Kim, H.-Y., Lee, K., Kim, G. T., Duesberg, G. S., Hallam, T., Boland, J. J., Wang, J. J., Donegan, J. F., Grunlan, J. C., Moriarty, G., Shmeliov, A., Nicholls, R. J., Perkins, J. M., Grieveson, E. M., Theuwissen, K., McComb, D. W., Nellist, P. D., and Nicolosi, V., "Two-dimensional nanosheets produced by liquid exfoliation of layered materials," Science 331(6017), 568-571 (2011).

[52] Hernandez, Y., Nicolosi, V., Lotya, M., Blighe, F. M., Sun, Z., De, S., McGovern, I. T., Holland, B., Byrne, M., Gun'Ko, Y. K., Boland, J. J., Niraj, P., Duesberg, G., Krishnamurthy, S., Goodhue, R., Hutchison, J., Scardaci, V., Ferrari, A. C., and Coleman, J. N., "High-yield production of graphene by liquid-phase exfoliation of graphite," Nat. Nanotechnol. 3(9), 563-568 (2008).

[53] Coleman, J. N., "Liquid-phase exfoliation of nanotubes and graphene," Adv. Funct. Mater. 19(23), 36803695 (2009).

[54] Withers, F., Yang, H., Britnell, L., Rooney, A. P., Lewis, E., Felten, A., Woods, C. R., Sanchez Romaguera, V., Georgiou, T., Eckmann, A., Kim, Y. J., Yeates, S. G., Haigh, S. J., Geim, A. K., Novoselov, K. S., and Casiraghi, C., "Heterostructures produced from nanosheet-based inks," Nano Lett. 14(7), 3987-3992 (2014).

[55] Torrisi, F., Hasan, T., Wu, W., Sun, Z., Lombardo, A., Kulmala, T. S., Hsieh, G.-W., Jung, S., Bonaccorso, F., Paul, P. J., Chu, D., and Ferrari, A. C., "Inkjet-printed graphene electronics," ACS Nano 6(4), 29923006 (2012).

[56] Hasan, T., Torrisi, F., Sun, Z., Popa, D., Nicolosi, V., Privitera, G., Bonaccorso, F., and Ferrari, A. C., "Solution-phase exfoliation of graphite for ultrafast photonics," Phys. Status Solidi 247(11), 2953-2957 (2010). 
[57] Zhang, M., Howe, R. C. T., Woodward, R. I., Kelleher, E. J. R., Torrisi, F., Hu, G., Popov, S. V., Taylor, J. R., and Hasan, T., "Solution processed $\mathrm{MoS}_{2}$-PVA composite for sub-bandgap mode-locking of a wideband tunable ultrafast Er:fiber laser," Nano Res. 8(5), 1522-1534 (2015).

[58] Woodward, R. I., Kelleher, E. J., Runcorn, T. H., Popov, S. V., Torrisi, F., Howe, R. C. T., and Hasan, T., "Q-switched fiber laser with $\mathrm{MoS}_{2}$ saturable absorber," in [CLEO 2014], SM3H.6, OSA, Washington, D.C. (2014).

[59] Woodward, R. I., Kelleher, E. J. R., Howe, R. C. T., Hu, G., Torrisi, F., Hasan, T., Popov, S. V., and Taylor, J. R., "Tunable Q-switched fiber laser based on saturable edge-state absorption in few-layer molybdenum disulfide $\left(\mathrm{MoS}_{2}\right)$," Opt. Express 22(25), 31113-31122 (2014).

[60] Woodward, R. I., Howe, R. C. T., Runcorn, T. H., Hu, G., Torrisi, F., Kelleher, E. J. R., and Hasan, T., "Wideband saturable absorption in few-layer molybdenum diselenide $\left(\mathrm{MoSe}_{2}\right)$ for Q-switching $\mathrm{Yb}$-, Erand Tm-doped fiber lasers," Opt. Express 23(15), 20051 (2015).

[61] Stankovich, S., Dikin, D. A., Dommett, G. H. B., Kohlhaas, K. M., Zimney, E. J., Stach, E. A., Piner, R. D., Nguyen, S. T., and Ruoff, R. S., "Graphene-based composite materials," Nature 442(7100), 282-6 (2006).

[62] Joensen, P., Frindt, R., and Morrison, S., "Single-layer $\mathrm{MoS}_{2}$," Mater. Res. Bull. 21(4), 457-461 (1986).

[63] Dresselhaus, M. S. and Dresselhaus, G., "Intercalation compounds of graphite," Adv. Phys. 51(1), 1-186 (2002).

[64] Eda, G., Yamaguchi, H., Voiry, D., Fujita, T., Chen, M., and Chhowalla, M., "Photoluminescence from chemically exfoliated $\mathrm{MoS}_{2}, "$ Nano Lett. 11(12), 5111-6 (2011).

[65] Zeng, Z., Yin, Z., Huang, X., Li, H., He, Q., Lu, G., Boey, F., and Zhang, H., "Single-layer semiconducting nanosheets: high-yield preparation and device fabrication," Angew. Chemie 50(47), 11093-7 (2011).

[66] Parvez, K., Li, R., Puniredd, S. R., Hernandez, Y., Hinkel, F., Wang, S., Feng, X., and Müllen, K., "Electrochemically exfoliated graphene as solution-processable, highly conductive electrodes for organic electronics," ACS Nano 7(4), 3598-606 (2013).

[67] Xia, Z. Y., Pezzini, S., Treossi, E., Giambastiani, G., Corticelli, F., Morandi, V., Zanelli, A., Bellani, V., and Palermo, V., "The exfoliation of graphene in liquids by electrochemical, chemical, and sonicationassisted techniques: A nanoscale study," Adv. Funct. Mater. 23(37), 4684-4693 (2013).

[68] Park, S. and Ruoff, R. S., "Chemical methods for the production of graphenes," Nat. Nanotechnol. 4(4), 217-24 (2009).

[69] Stankovich, S., Dikin, D. A., Piner, R. D., Kohlhaas, K. A., Kleinhammes, A., Jia, Y., Wu, Y., Nguyen, S. T., and Ruoff, R. S., "Synthesis of graphene-based nanosheets via chemical reduction of exfoliated graphite oxide," Carbon 45(7), 1558-1565 (2007).

[70] Eda, G. and Chhowalla, M., "Chemically derived graphene oxide: towards large-area thin-film electronics and optoelectronics," Adv. Mater. 22(22), 2392-415 (2010).

[71] Eda, G., Fanchini, G., and Chhowalla, M., "Large-area ultrathin films of reduced graphene oxide as a transparent and flexible electronic material," Nat. Nanotechnol. 3(5), 270-4 (2008).

[72] Paton, K. R., Varrla, E., Backes, C., Smith, R. J., Khan, U., O’Neill, A., Boland, C., Lotya, M., Istrate, O. M., King, P., Higgins, T., Barwich, S., May, P., Puczkarski, P., Ahmed, I., Moebius, M., Pettersson, H., Long, E., Coelho, J. a., O'Brien, S. E., McGuire, E. K., Sanchez, B. M., Duesberg, G. S., McEvoy, N., Pennycook, T. J., Downing, C., Crossley, A., Nicolosi, V., and Coleman, J. N., "Scalable production of large quantities of defect-free few-layer graphene by shear exfoliation in liquids," Nat. Mater. 13(6), 624-30 (2014).

[73] Mason, T. J. and Lorimer, J. P., [Applied Sonochemistry], Wiley-VCH Verlag GmbH and Co. KGaA, Weinheim, FRG (2002).

[74] Coleman, J. N., "Liquid exfoliation of defect-free graphene.," Acc. Chem. Res. 46(1), 14-22 (2013).

[75] Sun, Z., James, D. K., and Tour, J. M., "Graphene chemistry synthesis and manipulation," J. Phys. Chem. Lett. 2(19), 2425-2432 (2011).

[76] Zhi, L. and Müllen, K., "A bottom-up approach from molecular nanographenes to unconventional carbon materials," J. Mater. Chem. 18(13), 1472 (2008). 
[77] Peng, Y., Meng, Z., Zhong, C., Lu, J., Yu, W., Jia, Y., and Qian, Y., "Hydrothermal synthesis and characterization of single molecular layer $\mathrm{MoS}_{2}$ and $\mathrm{MoSe}_{2}, "$ Chem. Lett. 30(8), 772-773 (2001).

[78] Ramakrishna Matte, H. S. S., Gomathi, A., Manna, A. K., Late, D. J., Datta, R., Pati, S. K., and Rao, C. N. R., "MoS 2 and $\mathrm{WS}_{2}$ analogues of graphene," Angew. Chemie 49(24), 4059-4062 (2010).

[79] Matte, H. S. S. R., Plowman, B., Datta, R., and Rao, C. N. R., "Graphene analogues of layered metal selenides," Dalt. Trans. 40(40), 10322 (2011).

[80] Secor, E. B., Prabhumirashi, P. L., Puntambekar, K., Geier, M. L., and Hersam, M. C., "Inkjet printing of high conductivity, flexible graphene patterns," J. Phys. Chem. Lett. 4(8), 1347-1351 (2013).

[81] Stankovich, S., Piner, R. D., Nguyen, S. T., and Ruoff, R. S., "Synthesis and exfoliation of isocyanatetreated graphene oxide nanoplatelets," Carbon 44(15), 3342-3347 (2006).

[82] Zhang, M., Hu, G., Hu, G., Howe, R. C. T., Chen, L., Zheng, Z., and Hasan, T., "Yb- and Er-doped fiber laser Q-switched with an optically uniform, broadband $\mathrm{WS}_{2}$ saturable absorber," arXiv $\mathbf{1 5 0 7 . 0 3 1 8 8}$ (2015).

[83] Bae, S., Kim, S. J., Shin, D., Ahn, J.-H., and Hong, B. H., "Towards industrial applications of graphene electrodes," Phys. Scr. T146, 014024 (2012).

[84] Ho, X. and Wei, J., "Films of carbon nanomaterials for transparent conductors," Materials 6(6), 2155-2181 (2013).

[85] Zhu, Y., Sun, Z., Yan, Z., Jin, Z., and Tour, J. M., "Rational design of hybrid graphene films for highperformance transparent electrodes," ACS Nano 5(8), 6472-9 (2011).

[86] De, S., King, P. J., Lotya, M., O’Neill, A., Doherty, E. M., Hernandez, Y., Duesberg, G. S., and Coleman, J. N., "Flexible, transparent, conducting films of randomly stacked graphene from surfactant-stabilized, oxide-free graphene dispersions," Small 6(3), 458-64 (2010).

[87] Hecht, D. S., Hu, L., and Irvin, G., "Emerging transparent electrodes based on thin films of carbon nanotubes, graphene, and metallic nanostructures," Adv. Mater. 23(13), 1482-1513 (2011).

[88] Tung, V. C., Chen, L.-M., Allen, M. J., Wassei, J. K., Nelson, K., Kaner, R. B., and Yang, Y., "Lowtemperature solution processing of graphene-carbon nanotube hybrid materials for high-performance transparent conductors," Nano Lett. 9(5), 1949-1955 (2009).

[89] Kim, Y.-K. and Min, D.-H., "Durable large-area thin films of graphene/carbon nanotube double layers as a transparent electrode," Langmuir 25(19), 11302-11306 (2009).

[90] Tracton, A. A., ed., [Coatings Technology Handbook], CRC Press, Inc., Boca Raton FL (2006).

[91] Hutchings, I. M. and Martin, G. D., [Inkjet Technology for Digital Fabrication], John Wiley and Sons (2012).

[92] Jang, D., Kim, D., and Moon, J., "Influence of fluid physical properties on ink-jet printability," Langmuir 25(5), 2629-35 (2009).

[93] Derby, B., "Inkjet printing of functional and structural materials: fluid property requirements, feature stability, and resolution," Annu. Rev. Mater. Res. 40(1), 395-414 (2010).

[94] Fromm, J. E., "Numerical calculation of the fluid dynamics of drop-on-demand jets," IBM J. Res. Dev. 28(3), 322-333 (1984).

[95] Shin, P., Sung, J., and Lee, M. H., "Control of droplet formation for low viscosity fluid by double waveforms applied to a piezoelectric inkjet nozzle," Microelectron. Reliab. 51(4), 797-804 (2011).

[96] Hasan, T., Tan, P. H., Bonaccorso, F., Rozhin, A. G., Scardaci, V., Milne, W. I., and Ferrari, A. C., "Polymer-assisted isolation of single wall carbon nanotubes in organic solvents for optical-quality nanotube - polymer composites," J. Phys. Chem. C 112(51), 20227-20232 (2008).

[97] Howe, R. C. T., Woodward, R. I., Hu, G., Yang, Z., Kelleher, E. J. R., and Hasan, T., "Surfactantaided exfoliation of molydenum disulphide for ultrafast pulse generation through edge-state saturable absorption," Phys. Status Solidi In Press (2015).

[98] Steen, W. and Mazumder, J., [Laser Material Processing], Springer (2010).

[99] Dausinger, F., Lichtner, F., and Lubatschowski, H., [Femtosecond Technology for Technical and Medical Applications], Springer (2004).

[100] Hasan, T., Sun, Z., Wang, F., Bonaccorso, F., Tan, P. H., Rozhin, A. G., and Ferrari, A. C., "Nanotubepolymer composites for ultrafast photonics," Adv. Mater. 21(38), 3874-3899 (2009). 
[101] Martinez, A. and Sun, Z., "Nanotube and graphene saturable absorbers for fibre lasers," Nat. Photonics 7(11), 842-845 (2013).

[102] Keller, U., "Recent developments in compact ultrafast lasers," Nature 424(6950), 831-8 (2003).

[103] Svelto, O., [Principles of Lasers], Springer, 5th ed. (2010).

[104] Sun, Z., Hasan, T., Torrisi, F., Popa, D., Privitera, G., Wang, F., Bonaccorso, F., Basko, D. M., and Ferrari, A. C., "Graphene Mode-Locked Ultrafast Laser," ACS Nano 4(2), 803-810 (2010).

[105] Bao, Q., Zhang, H., Wang, Y., Ni, Z., Yan, Y., Shen, Z. X., Loh, K. P., and Tang, D. Y., "Atomic-layer graphene as a saturable absorber for ultrafast pulsed lasers," Adv. Funct. Mater. 19(19), 3077-3083 (2009).

[106] Wang, K., Wang, J., Fan, J., Lotya, M., O'Neill, A., Fox, D., Feng, Y., Zhang, X., Jiang, B., Zhao, Q., Zhang, H., Coleman, J. N., Zhang, L., and Blau, W. J., "Ultrafast saturable absorption of two-dimensional $\mathrm{MoS}_{2}$ nanosheets," ACS Nano 7(10), 9260-7 (2013).

[107] Li, D., Jussila, H., Karvonen, L., Ye, G., Lipsanen, H., Chen, X., and Sun, Z., "Ultrafast pulse generation with black phosphorus," arXiv $\mathbf{1 5 0 5 . 0 0 4 8 0}$ (2015).

[108] Lu, S. B., Miao, L. L., Guo, Z. N., Qi, X., Zhao, C. J., Zhang, H., Wen, S. C., and Fan, D. Y., "Broadband nonlinear optical response in multi- layer black phosphorus: an emerging infrared and mid-infrared optical material," Opt. Express 23(9), 3370-3377 (2015).

[109] Huang, Y., Luo, Z., Li, Y., Zhong, M., Xu, B., Che, K., Xu, H., Cai, Z., Peng, J., and Weng, J., "Widelytunable, passively Q-switched erbium-doped fiber laser with few-layer $\mathrm{MoS}_{2}$ saturable absorber," Opt. Express 22(21), 25258 (2014).

[110] Luo, Z., Huang, Y., Zhong, M., Li, Y., Wu, J., Xu, B., Xu, H., Cai, Z., Peng, J., and Weng, J., "1, 1.5, and 2 $\mu \mathrm{m}$ fiber lasers Q-switched by a broadband few-layer $\mathrm{MoS}_{2}$ saturable absorber," J. Light. Technol. 32(24), 4679-4686 (2014).

[111] Liu, H., Luo, A.-P., Wang, F.-Z., Tang, R., Liu, M., Luo, Z.-C., Xu, W.-C., Zhao, C.-J., and Zhang, H., "Femtosecond pulse erbium-doped fiber laser by a few-layer $\mathrm{MoS}_{2}$ saturable absorber," Opt. Lett. 39(15), 4591 (2014).

[112] Woodward, R. I., Howe, R. C. T., Hu, G., Torrisi, F., Zhang, M., Hasan, T., and Kelleher, E. J. R., "Fewlayer $\mathrm{MoS}_{2}$ saturable absorbers for short-pulse laser technology: current status and future perspectives," Photonics Res. 3(2), A30 (2015).

[113] Roxlo, C. B., Chianelli, R. R., Deckman, H. W., Ruppert, A. F., and Wong, P. P., "Bulk and surface optical absorption in molybdenum disulfide," J. Vac. Sci. Technol. A 5(4), 555 (1987).

[114] Wu, K., Zhang, X., Wang, J., Li, X., and Chen, J., "WS ${ }_{2}$ as a saturable absorber for ultrafast photonic applications of mode-locked and Q-switched lasers," Opt. Express 23(9), 11453 (2015).

[115] Bonaccorso, F. and Sun, Z., "Solution processing of graphene, topological insulators and other 2d crystals for ultrafast photonics," Opt. Mater. Express 4(1), 63-78 (2014).

[116] Sun, Z., Hasan, T., and Ferrari, A. C., "Ultrafast lasers mode-locked by nanotubes and graphene," Phys. E 44(6), 1082-1091 (2012).

[117] Hu, G., Zhang, M., Chen, L., Zhu, X., Hu, G., Howe, R. C. T., Zhao, X., Zheng, Z., and Hasan, T., "Q-switched Yb-doped fiber laser with $\mathrm{WS}_{2}$ saturable absorber," in [Cleo-PR 2015], 25A3-4 (2015).

[118] Sun, Z., Popa, D., Hasan, T., Torrisi, F., Wang, F., Kelleher, E. J. R., Travers, J. C., Nicolosi, V., and Ferrari, A. C., "A stable, wideband tunable, near transform-limited, graphene-mode-locked, ultrafast laser," Nano Res. 3(9), 653-660 (2010).

[119] Popa, D., Sun, Z., Torrisi, F., Hasan, T., Wang, F., and Ferrari, A. C., "Sub 200fs pulse generation from a graphene mode-locked fiber laser," Appl. Phys. Lett. 97(20), 203106 (2010).

[120] Popa, D., Sun, Z., Hasan, T., Torrisi, F., Wang, F., and Ferrari, A. C., "Graphene Q-switched, tunable fiber laser," Appl. Phys. Lett. 98(7), 073106 (2011).

[121] Zhang, M., Kelleher, E. J. R., Torrisi, F., Sun, Z., Hasan, T., Popa, D., Wang, F., Ferrari, A. C., Popov, S. V., and Taylor, J. R., "Tm-doped fiber laser mode-locked by graphene-polymer composite.," Opt. Express 20(22), 25077-84 (2012). 